\title{
Comparison of midterm results of endovascular aneurysm repair for ruptured and elective abdominal aortic aneurysms
}

\author{
José Oliveira-Pinto, MD, ${ }^{a, b, c}$ Rita Soares-Ferreira, MD, ${ }^{a, d}$ Nelson F. G. Oliveira, MD, a,e \\ Frederico M. Bastos Gonçalves, MD, PhD, ${ }^{\text {a,d }}$ Sanne Hoeks, PhD, ${ }^{f}$ Marie Josee Van Rijn, MD, PhD, ${ }^{a}$ \\ Sander Ten Raa, MD, PhD, ${ }^{a}$ Armando Mansilha, MD, PhD, ${ }^{b, c}$ and Hence J. M. Verhagen, MD, PhD, ${ }^{a}$ \\ Rotterdam, The Netherlands; and Porto, Lisbon, and Azores, Portugal
}

\begin{abstract}
Objective: Endovascular aneurysm repair (EVAR) became an increasingly preferred modality for abdominal aortic aneurysm (AAA) repair both in elective AAA repair (el-EVAR) and EVAR of a ruptured AAA ( $r$-EVAR) setting. Ruptured AAAs usually have more hostile anatomies and less time for planning. Consequently, more complications may arise after r-EVAR. The purpose of this study was to compare mi-term outcomes between r-EVAR and el-EVAR.

Methods: A retrospective cohort analysis of patients undergoing EVAR from 2000 to 2015 at a tertiary institution was performed. Patients with previous aortic surgery, nonatherosclerotic AAA and isolated iliac aneurysms were excluded. In-hospital casualties or patients who were intraoperatively converted to open repair were also excluded. For the midterm outcome analysis, only patients with at least two postoperative examinations (a 30-day computed tomography scan and a second postoperative examination performed 6 months or later) were considered. The primary end point was freedom from aneurysm-related complications (a composite of type I or III endoleak, aneurysm sac growth, migration of more than $5 \mathrm{~mm}$, device integrity failure, AAA-related death, late postimplant rupture, or AAA-related secondary intervention). Freedom from secondary interventions, neck-related events (defined as a composite of type IA endoleak, migration of more than $5 \mathrm{~mm}$, or preemptive neck-related secondary intervention) and late survival were secondary end points. The impact of device instructions for use (IFU) compliance on neck events was also assessed.
\end{abstract}

Results: The study included 565 patients (65 r-EVAR and 500 el-EVAR). Eighty-two patients were treated outside proximal neck IFU, 13 in the r-EVAR group (21.3\%) and $69(14.5 \%)$ in the el-EVAR $(P=.16)$. During the index hospitalization, there were more complications (12.3\% vs 3.2\%; $P=.001$ ) and reinterventions (12.3\% vs $2.8 \%$; $P<.001$ ) in the r-EVAR group. After discharge, median clinical follow-up time was 4.3 years (interquartile range, 2.1-7.0 years) without differences between both groups. Five-year freedom from AAA-related complications was $53.9 \%$ in the r-EVAR group and $65.4 \%$ in the el-EVAR $(P=.21)$. In multivariable analysis the $r$-EVAR group was not at increased risk for late complications (hazard ratio [HR], 0.94; 95\% confidence interval [CI], 0.54-1.61; $P=.81$ ). Five-year freedom from neck-related events was 74\% in $r-E V A R$ and $82 \%$ in the el-EVAR group $(P=.345)$. Patients treated outside neck IFU were at greater risk for neck-related events both in r-EVAR (HR, 6.5; 95\% Cl, 1.8-22.9; $P=.004$ ) and el-EVAR group (HR, 2.6; 95\% Cl, 1.5-4.5; $P<.001)$. Freedom from secondary interventions at 5 years was $63.0 \%$ for $r$-EVAR and $76.9 \%$ for el-EVAR $(P=.16)$. Survival at 5 years was $68.8 \%$ in the r-EVAR group and $73.3 \%$ in the el-EVAR group $(P=.30)$.

Conclusions: Durable and sustainable midterm outcomes were found for both r-EVAR and el-EVAR patients who survived the postoperative period. Patients treated outside the IFU are at greater risk for late complications. Surveillance protocols may be tailored according to individual anatomy and IFU compliance rather than timing of repair. (J Vasc Surg 2019; :1-10.)

Keywords: Aortic aneurysm; Abdominal; Aneurysm; Ruptured aortic; Blood vessel prosthesis implantation; Midterm; Retrospective studies

\footnotetext{
From the Department of Vascular Surgery, Erasmus University Medical Centre, Rotterdama ; the Department of Angiology and Vascular Surgery, Centro Hospitalar São João, Porto ; the Department of Surgery and Physiology, Faculty of Medicine of Oporto, Portoc; the Department of Angiology and Vascular Surgery, Hospital de Santa Marta, Centro Hospitalar de Lisboa Central, Lisbond; the Department of Angiology and Vascular Surgery, Hospital do Divino Espirito Santo, Ponta Delgada, Azores' ; and the Department of Anesthesiology Erasmus University Medical Center, Rotterdam. ${ }^{f}$

Author conflict of interest: N.O.: Educational research grant from the Lijf en Leven Foundation; F.C.: Consultant for Medtronic and W. L. Gore \& Associates: S.H.: Educational research grant from the Lijf en Leven Foundation; H.V. Consultant for Medtronic, USA, W. L. Gore \& Associates, USA, Endologix, USA, and Arsenal AAA, USA.
}

Additional material for this article may be found online at www.jvascsurg.org. Correspondence: José Oliveira-Pinto, MD, Department of Vascular Surgery, Erasmus University Medical Centre, Rotterdam, s-Gravendijkwal 2303015 CE, Rotterdam, The Netherlands (e-mail: oliveirapintoj89@gmail.com).

The editors and reviewers of this article have no relevant financial relationships to disclose per the JVS policy that requires reviewers to decline review of any manuscript for which they may have a conflict of interest. 0741-5214

Copyright $\odot 2019$ by the Society for Vascular Surgery. Published by Elsevier Inc. https://doi.org/10.1016/j.jvs.2019.07.091 
Endovascular aneurysm repair for ruptured aortic abdominal aneurysms ( $r$-EVAR) has been increasingly used owing to its potential short-term benefits on survival and morbidity. ${ }^{1.2}$ However, the possibility of developing aneurysm-related complications remains the main drawback and lifelong surveillance is therefore warranted. ${ }^{3}$ Previous data suggest that patients who survive the perioperative period following $r$-EVAR may have similar long-term survival rates when compared with patients undergoing elective EVAR (el-EVAR). ${ }^{4}$ However, r-EVAR is frequently performed in more hostile anatomy, with less time to plan and endograft selection is limited by the available stock. ${ }^{5}$ Consequently, these patients may be at greater risk of developing complications than those treated electively. Although there are some reports comparing outcomes following r-EVAR and el-EVAR, data regarding longer term follow-up remains scarce. ${ }^{6,7}$ The purpose of this study was to compare midterm outcomes between $r$-EVAR and el-EVAR in patients surviving in-hospital stay.

\section{METHODS}

Design and population. A retrospective cohort study was designed based on a prospectively maintained database, including all patients undergoing standard EVAR in a single tertiary referral center in The Netherlands. Informed consent was not required according to institutional policy on retrospective research. All consecutive patients undergoing EVAR between January 2000 and December 2015 for infrarenal AAA were included. Patients with previous aortic surgery or with a diagnosis other than degenerative AAA (ie, anastomotic, infectious, or isolated iliac aneurysms) were excluded. Additionally, patients who died in the perioperative period (defined as death during the index admission) or who were intraoperatively converted to open repair were excluded from the analysis. Because our population usually performs first postoperative computed tomography angiography (CTA) during admission, only patients with at least two postoperative imaging examinations (a first 30-day contrast-enhanced CTA and a second examination, performed more than 6 months after EVAR), were considered for this midterm follow-up analysis.

Postoperative surveillance. At the beginning of the study period, CTA was performed at 1, 6, and 12 months, and yearly thereafter. However, the 6-month CTA has been progressively reserved for selected patients with or at an increased risk of developing aneurysm-related complications. Alternatively, if patients were considered by the treating physician to be at low risk of complications or had renal function impairment, color duplex ultrasound examination or a noncontrast CT scan was preferred during follow-up. Upon the detection of an adverse event on these imaging modalities, such as enlargement of more than $5 \mathrm{~mm}$ or an endoleak other than a type II endoleak, the patient would undergo CTA.

\section{ARTICLE HIGHLIGHTS}

Type of Research: Retrospective analysis of a prospectively collected single-center database

Key Findings: Comparison of outcome of 565 early survivors of endovascular aneurysm repair (EVAR) for ruptured $(n=65)$ and elective $(n=500)$ abdominal aortic aneurysms who had at least two postoperative imaging examinations revealed durable results in both groups, despite more hostile anatomy and less time for planning after ruptured EVAR. No differences at baseline were found regarding sealing length and instructions for use adherence.

Take Home Message: This study suggests that durable results can be reached in early survivors after both ruptured and elective EVAR as long as instructions for use are respected and proper sealing is obtained.

Data management. Baseline demographics and anatomic measurement were collected at the time of the intervention. All subsequent follow-up data were prospectively obtained upon outpatient visits and/or patient record consult at regular, predefined intervals. Date of death was retrieved from hospital records and from the Dutch Center Bureau of Statistics.

Image analysis and measurements. All measurements (diameters, lengths, angles, and volumes) were obtained on CT imaging using semiautomatically generated center lumen line reconstructions performed on dedicated reconstruction software (3mensio Vascular 4.2; Medical Imaging B.V., Bilthoven, The Netherlands). All imaging data were acquired by three observers with experience in image analysis (J.O.P., N.O., F.B.G.).

Proximal and distal sealing lengths were measured on the first postoperative CTA (30-day CTA). The sealing length was considered to be the distance where the entire circumference of the aortic and iliac vessel walls and the endograft are completely adjacent. The method of sealing length measurement has been described elsewhere. ${ }^{8}$ Sufficient postoperative seal was considered if a minimum seal length of $10 \mathrm{~mm}$ was measured proximally and distally according to previous reports. ${ }^{8,9}$

In a previous report, our group has demonstrated high rates of interobserver agreement regarding aneurysm diameter, neck diameter, neck length, and proximal seal length measurements with this method. ${ }^{8,10}$ Aneurysm volume and neck angulation were also measured according to previously validated method. ${ }^{17,12}$

Aneurysm neck length was defined as the distance from the lowermost renal artery to the level where the aortic diameter increases by more than $10 \%$. Aneurysm neck thrombus and calcification were categorized according to infrarenal aortic neck circumferential 
involvement. Neck configuration was classified according to published methodology. ${ }^{13}$

After scrutiny of the preoperative CT scan, compliance with the device manufacturers' instructions for use (IFU) for the specific device implanted was assessed.

Definitions. Patient comorbidities and aneurysmrelated outcomes were reported according to the recommendations from the Society for Vascular Surgery/ American Association of Vascular Surgery ad hoc Committee for Standardized Reporting Practices in Vascular Surgery..$^{14}$ An AAA was considered ruptured when evidence of retroperitoneal bleeding was present on the preoperative CTA. Intraoperative complications were considered to have occurred if the device was not deployed at the intended position, if type I or III endoleak or graft obstruction was present, or if unplanned endovascular or surgical procedures were necessary. Complications during the index hospitalization (or $<30$ days) were defined as type I or III endoleak, graft obstruction/ limb ischemia, or if unplanned endovascular or surgical procedures were necessary within this timeframe.

AAA-related complications were defined as a composite of the following: direct (type I or III) endoleak, aneurysm sac expansion or more than $5 \%$ in volume or $5 \mathrm{~mm}$ in diameter, migration of more than $5 \mathrm{~mm}$, graft infection or thrombosis, device integrity failure, AAA-related death, late postimplant rupture, or any AAA-related secondary intervention. Secondary interventions were considered to be AAA related if performed to resolve or prevent a possible complication associated with the initial treatment of the AAA and included proximal cuff and stent implant, distal extension, catheter-based thrombolysis, iliac angioplasty, coil or glue embolization of aortic branch vessels, balloon thrombectomy, femoral-femoral crossover, conversion to open repair, and open or laparoscopic ligation of collaterals.

Sac growth was defined as a more than $5 \%$ increase in aneurysm sac volume or as a more than $5 \mathrm{~mm}$ increase in sac diameter. A neck-related event was defined as a composite of type IA endoleak, neck-related secondary intervention, or migration of more than $5 \mathrm{~mm}$.

End points. The primary end point was freedom from AAA-related complications. Individual components of the composite outcome were used as secondary end points: freedom from secondary intervention, sac growth, types I and III endoleak, device migration of more than $5 \mathrm{~mm}$, conversion to open repair, and AAA-related death or AAA rupture. Additionally, neck-related events and overall mortality were also assessed. Impact on neck IFU compliance on neck event was also analyzed.

Statistical analysis. Categorical variables are presented as count and percentage, and compared using the Pearson's $\chi^{2}$ test. Continuous variables are presented as mean and standard deviation or as median and interquartile range (IQR). Differences between groups were analyzed using the Mann-Whitney $U$ test for independent samples with non-normal distributions or with the Student's $t$-test and significance with the independent samples test for nonrelated variables with normal distributions.

Survival curves for freedom from aneurysm-related adverse events were estimated by Kaplan-Meier methods, and equality was evaluated with the MantelCox log-rank test. Aneurysm-related outcome variables were assessed by Cox hazards regression models. Age and gender were included a priori in the Cox model. Multivariate regression was performed adjusting for baseline clinical and morphologic features differently distributed among groups at a $P$ value of less than .1 level. Confidence intervals (Cls) of 95\% were used and statistical significance was considered if the $P$ was less than .05. All statistical analysis was performed using SPSS 24.0 (IBM, Armonk, NY).

\section{RESULTS}

From January 2000 to December 2015, 660 patients underwent standard EVAR. Sixty-four patients were considered not eligible for this study, including 33 anastomotic or other pseudoaneurysms, 21 isolated iliac aneurysms, 9 infectious aneurysms, and 1 traumatic rupture.

Among the remaining study population of 596 patients (89 r-EVAR and 507 el-EVAR), 26 patients died during the index hospitalization (22 r-EVAR and 4 el-EVAR) and 5 patients were intraoperatively converted to open repair (4 r-EVAR and 1 el-EVAR), leaving a final study population of 565 patients, 65 r-EVAR (11.5\%) and 500 el-EVAR $(88.5 \%)$ patients. Mean age was $73.2 \pm 7.6$ years and $89.9 \%$ were male. Baseline demographics are presented in Table I.

Baseline anatomic features were distinct among groups. In the r-EVAR group, the aneurysms were larger (median diameter, $76 \mathrm{~mm}$ [IQR, $62-89 \mathrm{~mm}$ ], vs $60 \mathrm{~mm}$ [IQR, 54-68 mm]; $P<.001$ ), the proximal necks were shorter (neck $<15 \mathrm{~mm}: 21.3 \%$ vs $8.6 \% ; P=.002$ ) and more angulated ( $\alpha$ angle $>45^{\circ}: 21.3 \%$ vs $10.3 \% ; P=.011$ $\beta$-angle $>60^{\circ}: 31.1 \%$ vs $17.4 \% ; P=.010$ ). Among the r-EVAR group, a higher proportion of patients were treated using aortouni-iliac devices $(33.3 \%$ vs $3.2 \%$; $P<.001$; Table I).

A preoperative $C T$ scan of adequate quality was missing in 27 patients ( 4 in r-EVAR and 23 in el-EVAR). Eighty-two patients were treated outside proximal neck IFU, 13 $(21.3 \%)$ in the r-EVAR group and $69(14.5 \%)$ in the el-EVAR $(P=.16)$. Among patients outside neck IFU, in 16 (20\%) IFU violation was owing to neck length (2 r-EVAR and 14 el-EVAR) and 66 (80\%) patients owing to neck angulation (11 r-EVAR and 55 el-EVAR).

Proximal and distal sealing of $10 \mathrm{~mm}$ or greater at the 30-day CT imaging was found in $44 \mathrm{r}$-EVAR patients (74.6\%) and in 360 el-EVAR patients (77.8\%) $(P=.84)$ 
Table I. Baseline demographic and morphological characteristics

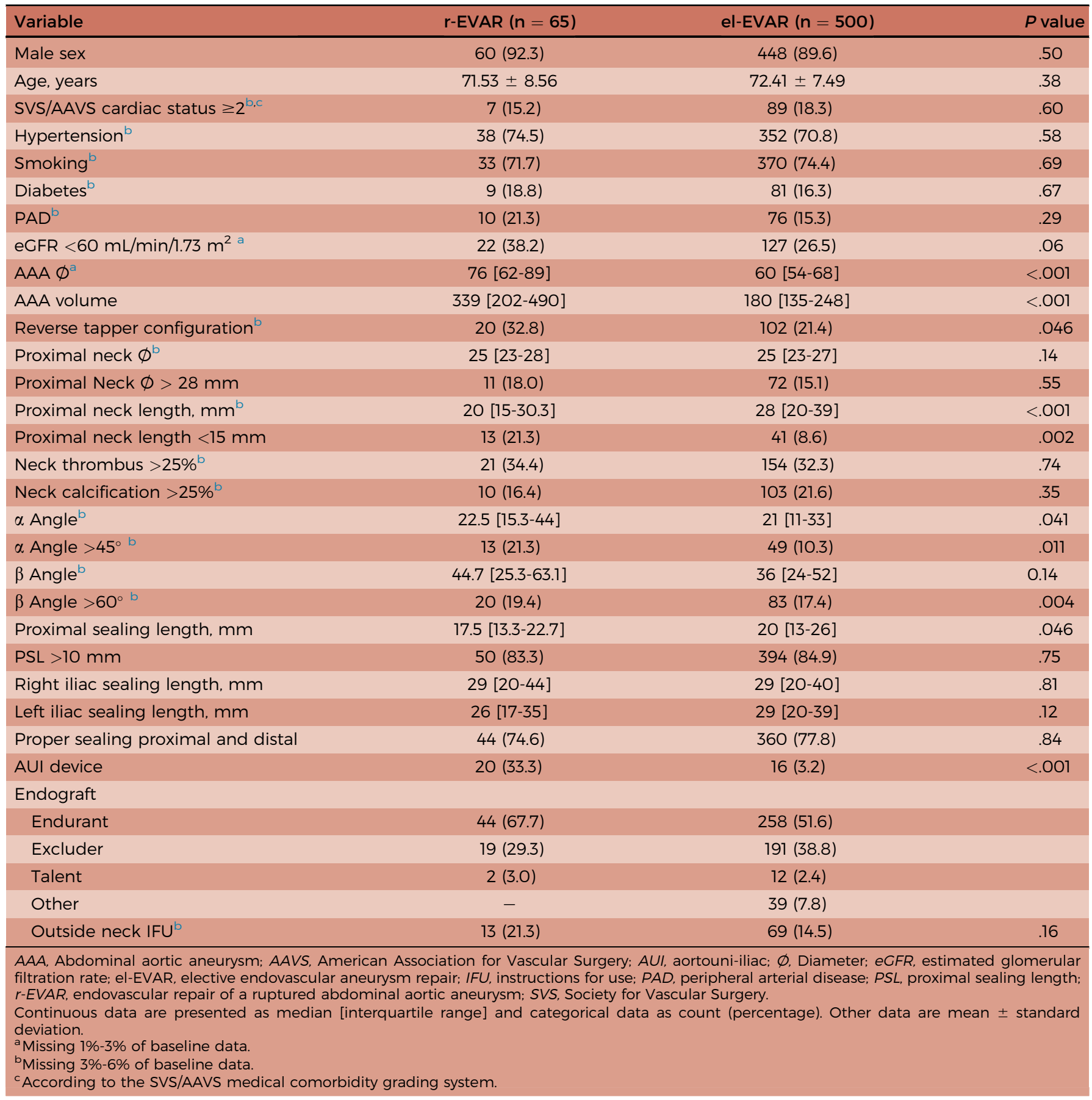

Intraoperative and in-hospital outcomes. Generally, there was no difference among groups regarding intraoperative complications (13.8\% r-EVAR vs 10.6\% el-EVAR; $P=.43$ ). There were three intraoperative type IA endoleaks in the r-EVAR group, all of them solved with proximal cuff placement. Twenty-six intraoperative type IA endoleak were observed in the el-EVAR group: 16 of them needed a proximal extension, 7 patients solved with ballooning only, and in 3 patients a small type IA persisted and were kept under surveillance.
During the index hospitalization, more complications (12.3\% vs 3.2\%; $P=.001$ ) and secondary interventions (12.3\% vs $2.8 \% ; P=.001$ ) were observed in the $r$-EVAR group. Three r-EVAR patients needed decompressive laparotomies owing to abdominal compartment syndrome. Two r-EVAR patients and three el-EVAR had a type IA endoleak detected in the postoperative CT scan and both underwent proximal cuff extension. One 88-year-old el-EVAR patient had a small type IA endoleak detected but was kept under strict surveillance. 
Table II. Intraoperative and in-hospital outcomes

\begin{tabular}{lccc}
\hline & r-EVAR $(\mathbf{n}=65)$ & el-EVAR $(\mathbf{n}=\mathbf{5 0 0})$ & $P$ value \\
\hline Intraoperative complication & $9(13.8)$ & $53(10.6)$ & .43 \\
\hline Type IA endoleak & $3(4.6)$ & $26(5.2)$ & .84 \\
Type IB endoleak & $2(3.1)$ & $6(1.2)$ & .23 \\
Type III endoleak & $0(0)$ & $1(.2)$ & .72 \\
Other & $2(3.1)$ & $18(3.6)$ & .83 \\
Intraoperative unexpected interventions & $8(12.3)$ & $38(7.6)$ & .19 \\
\hline Proximal cuff & $3(4.4)$ & $22(4.6)$ & .94 \\
\hline Distal extension & $4(6.2)$ & $6(1.2)$ & .004 \\
Conversion to AUI & $1(1.5)$ & $2(0.4)$ & .24 \\
Renal stent & $0(0)$ & $4(.8)$ & .47 \\
\hline Iliac PTA & $1(1.5)$ & $6(1.2)$ & .039 \\
Complications at 30 days & $8(12.3)$ & $16(3.2)$ & .001 \\
\hline Type la & $2(3.1)$ & $4(.8)$ & .09 \\
\hline Limb ischemia & $1(1.5)$ & $8(1.4)$ & .97 \\
Other & $4(6.2)$ & $1(1.2)$ & $<.001$ \\
\hline Reinterventions at 30 days, patients & $8(12.3)$ & $14(2.8)$ & $<.001$ \\
\hline Proximal cuff & $1(1.5)$ & $4(.6)$ & .39 \\
\hline Distal extension & $1(1.5)$ & $0(0)$ & .006 \\
\hline Thrombectomy/PTA & $1(1.5)$ & $8(1.6)$ & .97 \\
\hline Other & $5(7.4)$ & $4(.8)$ & $<.001$ \\
\hline AUI, Aortouni-iliac; el-EVAR, elective endovascular aneurysm repair; $r$-EVAR, endovascular repair of a ruptured abdominal aortic aneurysm; PTA, \\
percutaneous transluminal angioplasty. \\
Dichotomous data are presented as count (\%).
\end{tabular}

Additionally, two patients (one r-EVAR and one el-EVAR) had an asymptomatic limb occlusion, which was visualized in the postoperative CTA; both underwent thrombolysis and subsequent distal extension. In the el-EVAR group, partial overstenting of the renal artery was noted and the patient underwent renal stenting to avoid a subsequent occlusion. Finally, limb compression at the aortic bifurcation level was identified in one patient and subsequent percutaneous transluminal angioplasty was performed. Detailed data of intraoperative and perioperative outcomes are presented in Table II.

Aneurysm-related complications. Two postoperative imaging examinations were available in 527 patients (60 r-EVAR and 467 el-EVAR) and these patients were accountable for midterm analysis. Thirty-eight patients did not have two postoperative examinations: 12 patients were followed elsewhere after EVAR, 22 patients died before performing second postoperative examination ( 4 r-EVAR [6.2\%] and 18 el-EVAR [3.6\%] patients) and 4 patients choose not to have further follow-up.

The median clinical follow-up was 4.3 years (IQR, 2.17.0 years) and was similar between groups ( $r$-EVAR, 4.0 years [IQR, 2.0-5.6 years] and el-EVAR, 4.2 years [IQR, 2.1-7.0 years]; $P=.29$ ) (Table III).

After discharge, 21 r-EVAR patients (35\%) had aneurysm-related complications and these occurred in
146 patients (31.3\%) in the el-EVAR group (Table III). The 3-year and 5-year freedom from AAA-related complications were $73.1 \%(n=24$; standard error [SE], 0.066) and $53.9 \%$ ( $n=13 ; \mathrm{SE}, 0.083$ ) in the r-EVAR group and $80.6 \%(n=233 ;$ SE, 0.020) and $65.4 \%(n=151 ; S E, 0.027)$ in the el-EVAR, respectively $(P=.21$ ) (Fig 1). Individual components of the latter outcome are reported in Table III. In an adjusted Cox proportional hazards model, the r-EVAR group was not at an increased risk for late complications (HR, 0.94; $95 \% \mathrm{Cl}, 0.54-1.61 ; P=.81)$ (Table IV).

Aneurysm sac growth occurred similarly between groups (11 r-EVAR [18.3\%] and 86 el-EVAR [18.4\%]; $P=$ .98). Postimplant ruptures occurred in eight patients, three r-EVAR patients (5.0\%) and five among el-EVAR (1.1\%; $P=.052)$, resulting in death in four patients, two (3.3\%) r-EVAR and two el-EVAR (0.4\%; $P=.015)$.

Eighty-five patients had neck-related events: $11 \mathrm{r}$-EVAR (18.3\%) and 74 el-EVAR (15.8\%) patients. The 3- and 5 -year freedom from neck-related events was 87\% ( $n=30 ;$ SE, 0.051) and $74 \%(n=; S E, 0.075)$ in r-EVAR and $90 \%(\mathrm{n}=272 ; \mathrm{SE}, 0.015)$ and $83 \%(\mathrm{n}=174 ; \mathrm{SE}$, 0.022 ) in the el-EVAR group $(P=.35$ ) (Fig 2$)$. There were no differences between $r$-EVAR and el-EVAR regarding type IA endoleak occurrence (3 [5.0\%] vs 32 [6.9\%]; $P=.59$ ), or migration (8 [13.8\%] vs 38 [8.3\%]; $P=.17$ ). Patients treated outside neck IFU were at greater risk 
Table III. Aneurysm-related complications after discharge

\begin{tabular}{|c|c|c|c|}
\hline Outcome measure & $r-\operatorname{EVAR}(n=60)$ & el-EVAR $(n=467)$ & $P$ value \\
\hline Follow-up, years & $4.0[2.0-5.6]$ & $4.2[2.1-7.0]$ & .29 \\
\hline Overall mortality & $25(41.7)$ & $182(39.0)$ & $.30^{\mathrm{a}}$ \\
\hline Aneurysm-related complications, patients & $21(35.0)$ & $146(31.3)$ & $.21^{a}$ \\
\hline Postimplant rupture & $3(5.0)$ & $5(1.1)$ & .052 \\
\hline Secondary endoleaks, patients & $13(21.7)$ & $123(26.3)$ & .436 \\
\hline Type IA & $3(5.0)$ & $32(6.9)$ & .59 \\
\hline Type IB & $2(3.3)$ & $22(4.7)$ & .63 \\
\hline Type II & $11(18.3)$ & $87(18.6)$ & .96 \\
\hline Type III & $1(1.7)$ & $4(0.9)$ & .54 \\
\hline Sac growth & $11(18.3)$ & $86(18.4)$ & .98 \\
\hline Migration $>5 \mathrm{~mm}$ & $8(13.8)$ & $38(8.3)$ & 17 \\
\hline Limb thrombosis & $4(6.7)$ & $14(3.0)$ & .14 \\
\hline Endograft infection & $\mathrm{O}(0)$ & $5(1.1)$ & .42 \\
\hline Secondary interventions, patients & $16(26.7)$ & $101(21.6)$ & $0.16^{\mathrm{a}}$ \\
\hline Proximal cuff/Palmaz stent & $4(6.7)$ & $30(6.4)$ & .94 \\
\hline Conversion AUI & $\mathrm{O}(0)$ & $3(0.6)$ & .53 \\
\hline Iliac PTA & $\mathrm{O}(0)$ & $4(0.9)$ & .47 \\
\hline Distal extension & $10(16.7)$ & $36(7.7)$ & .02 \\
\hline Thrombolysis + PTA & $1(1.7)$ & $6(1.3)$ & .80 \\
\hline Thrombectomy + limb extension/PTA & $1(1.7)$ & $7(1.5)$ & .92 \\
\hline Renal stenting & $\mathrm{O}(0)$ & $1(0.2)$ & .72 \\
\hline Open/laparoscopic ligation of collaterals & $\mathrm{O}(0)$ & $9(1.9)$ & .28 \\
\hline Percutaneous embolization of IMA/lumbars & $\mathrm{O}(0)$ & $11(2.4)$ & .23 \\
\hline Relining & $1(3.3)$ & $8(1.7)$ & .39 \\
\hline Open conversion & $5(8.3)$ & $18(3.9)$ & .11 \\
\hline
\end{tabular}

for neck-related events both in the r-EVAR group (HR, 6.5; $95 \% \mathrm{Cl}, 1.8-22.9 ; P=.004$ ] and in the el-EVAR group (HR, 2.6; $95 \% \mathrm{Cl}$, 1.5-4.5 $P<.001$ ).

Secondary interventions. After the index hospitalization for EVAR, 155 secondary interventions were performed in 117 patients (16 r-EVAR [26.7\%] vs 101 el-EVAR [21.6\%]). Freedom from secondary interventions at 3 and 5 years were $83.0 \%(n=27 ;$ SE, 0.054) and $63.0 \%(n=18$; SE, 0.081) for $r$-EVAR and 87.0\% $(n=263 ;$ SE, 0.017) and 76.9\% ( $\mathrm{n}=16$; SE, 0.025) for el-EVAR ( $P=.16$ ) (Fig 3). Detailed data regarding secondary interventions are presented in Table III.

In multivariable regression analysis, the risk of secondary intervention was not higher among r-EVAR patients (HR 0.97; 95\% Cl, 0.50-1.86; $P=.93$; Table IV). In the $r$-EVAR group, three patients were electively converted to open repair owing to type IA endoleak (one of them had a proximal cuff placed but type IA endoleak reoccurred). In the el-EVAR, group 26 patients were intervened owing to type IA endoleaks (16 proximal cuffs, 9 conversions to open repair, and 1 patient underwent relining with gaining of proximal seal). In six patients presenting with type IA endoleak, no procedure was carried out: one patient owing to spontaneous sealing, three patients owing to refusal for further intervention, and two patients owing to unsuitable anatomy for endovascular repair and unacceptable risk for open repair.

All-cause mortality and AAA-related mortality. During the study period, death beyond index hospitalization occurred in 207 (39.3\%) patients: 25 in the r-EVAR group (41.7\%) and 182 in el-EVAR group (39.0\%). Survival at 3 and 5 years was $76.6 \%(n=41 ; S E, 0.057)$ and $68.8 \%$ $(n=33 ; S E, 0.063)$ in the r-EVAR group and $84.4 \%(n=331$; SE, 0.018) and $73.3 \%(n=190 ; S E, 0.025)$, respectively, in the el-EVAR group, $(P=.30)$ (Fig 4). Overall survival including patients previously excluded owing to lack of two postoperative imaging examinations is shown in the Supplementary Fig (online only). Of these, nine deaths were AAA related, two in the r-EVAR group and seven in the el-EVAR group. In these patients, postimplant rupture was the cause in four patients (time frame of death: 1.9, 3.0, 3.3, and 8.0 years after EVAR), endograft 


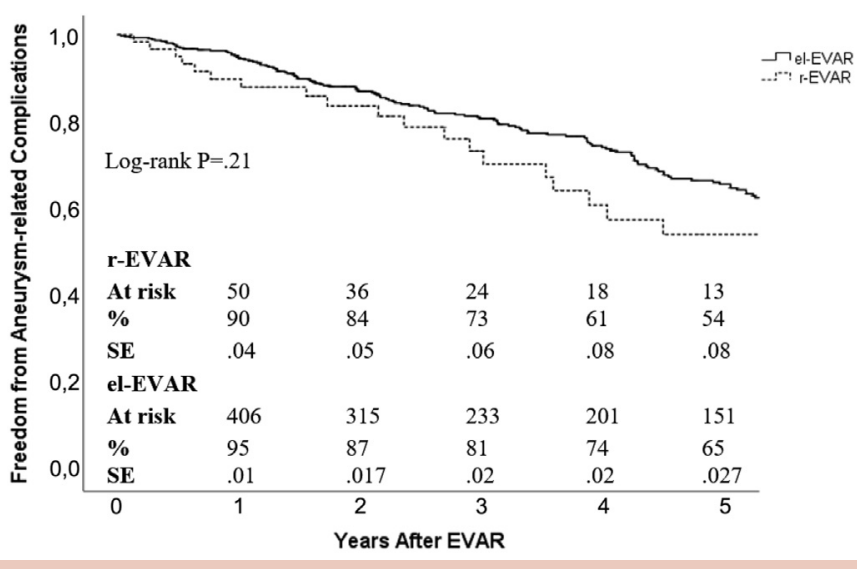

Fig 1. Kaplan-Meier curve for freedom from aneurysmrelated complications after endovascular repair of a ruptured abdominal aortic aneurysm ( $r$-EVAR) and elective endovascular aneurysm repair (el-EVAR). KaplanMeier curves only include data from patients with two postoperative examinations. SE, Standard error.

infection without rupture in two ( 2 and 3 years after EVAR), and owing to postoperative complications after a secondary intervention in three patients (at 3,10, and 15 years after EVAR). In multivariable analysis correcting for baseline differences, r-EVAR was not an independent predictor for late overall mortality $(\mathrm{HR}, 0.62 ; 95 \% \mathrm{Cl}$, 0.351.11; $P=.11$ ) (Table IV).

\section{DISCUSSION}

Patients with patients with a ruptured AAA have more complex anatomies and require an expeditious preoperative planning. Therefore, a theoretical greater risk of complications might be anticipated after r-EVAR after the initial hospitalization period. Our results suggest that, excluding the early perioperative period, sustainable midterm outcomes can be achieved in both ruptured and elective settings.

Comparative data on late outcomes following r-EVAR and el-EVAR is scarce. Bastos Gonçalves et al ${ }^{15}$ had previously reported that r-EVAR was not a significant risk factor for late complications when compared with el-EVAR (HR, 0.87; 95\% Cl, 0.43-1.79; $P=.71) .^{15}$ Although some patients figuring in this multicentric report are also included in our current study, other patients treated at our center were added as well as follow-up was extended. Furthermore, our r-EVAR group was compared with an el-EVAR group obtained from the same population treated by the same surgical team during the same study period. However, this factor may limit the generalizability of the presented results to other centers, the risk of bias inherent to the repair itself as well as differences in the study population's genetic background is thus reduced in our analyses. Also Broos et al ${ }^{6}$ found no differences regarding secondary interventions among $r$-EVAR and el-EVAR patients in a single-center report including 863 patients (90 r-EVAR with a median follow-up of 21 months and 773 el-EVAR patients with a median follow-up of 38 months). At first glance, our results are in agreement with these authors. Still, in this study a there was a tendency toward higher reintervention rates was observed in r-EVAR group (21.8\% vs $15.8 \%$ at 5 years; $P=.064$ ). However, an adjusted analysis is not provided, which may limit conclusions regarding timing of repair and incidence of secondary interventions. Even though baseline anatomy was significantly more hostile among r-EVAR patients in our cohort, no difference was found regarding neck IFU compliance between groups. Additionally, in our cohort, the number of patients with more than $10 \mathrm{~mm}$ of proximal and distal seal on the initial postoperative seal was also similar between groups. Previous reports have demonstrated that absence of endoleak at 30 days in conjunction with adequate proximal and distal seal represent a reliable proxy of a low risk of complications, at least up to 5 years after the intervention, ${ }^{8,9}$ and that it may be safe to have a less stringent surveillance program for this patient group. Consequently, we hypothesized that if those two conditions are respected, r-EVAR patients may follow the same less stringent surveillance. However it is important to note that both freedom from AAArelated complications and freedom from secondary in terventions seem to have decreased more abruptly among the r-EVAR cohort from 3 years onward, although these differences were not statistically significant; however, they are potentially clinically relevant.

We consider that our study's sample size and the available follow-up may have limited the power to detect a statistical difference and thus advise caution when interpreting our results. A prospective study, designed as a noninferiority study and with adequate power calculation, would add strength to the conclusion. More studies with longer term follow-up are needed to safely advice on the possibilities of changing the intensity of surveillance programs after $r$-EVAR. Still, after multivariable analysis, correcting for baseline differences among groups, in an attempt to isolate the effect of timing of repair no greater risk was found for r-EVAR patients (HR, 0.94; 95\% Cl, 0.54-1.61; $P=.81$ ). Our study is in line with the 3-year results of the IMPROVE Trial reporting $21 \%$ of patients $(26 / 125$, per protocol analysis) undergoing reintervention between 90 days and 3 years after EVAR, Secondary intervention rates we report here may seem slightly higher to the IMPROVE trial, but our cohort was followed for a longer period of time. Additionally, in our study only AAA-related reinterventions are reported, as defined by Society for Vascular Surgery reporting standards, in the IMPROVE trial also reinterventions not graft-related were considered. ${ }^{14,16,17}$

However, using the same 3-year cutoff in our study, freedom from secondary interventions was $83 \%(n=27)$ which is similar to described the IMPROVE trial: 38 reinterventions in 26 of 125 patients who 
Table IV. Multivariable analysis for aneurysm-related complications, secondary interventions and overall mortality

\begin{tabular}{|c|c|c|c|c|c|c|c|c|c|}
\hline & \multicolumn{3}{|c|}{$\begin{array}{l}\text { Aneurysm-related } \\
\text { complications }\end{array}$} & \multicolumn{3}{|c|}{ Secondary interventions } & \multicolumn{3}{|c|}{ Overall mortality } \\
\hline & $\mathrm{HR}$ & $95 \% \mathrm{Cl}$ & $P$ value & $\mathrm{HR}$ & $95 \% \mathrm{Cl}$ & $P$ value & HR & $95 \% \mathrm{Cl}$ & $P$ value \\
\hline Male sex & .91 & $.55-1.50$ & .72 & .90 & $.49-1.66$ & .75 & 1.14 & $.72-1.80$ & .58 \\
\hline eGFR $<60 \mathrm{~mL} / \mathrm{min} / 1.73 \mathrm{~m}$ & .56 & $.36-.85$ & .008 & .50 & $.29-.86$ & .01 & 1.72 & $1.26-2.36$ & .001 \\
\hline$\beta$ Angle $>60^{\circ}$ & 1.30 & $.85-1.99$ & .22 & 1.26 & $.75-2.13$ & .39 & .80 & $.51-1.25$ & .33 \\
\hline Neck length $<15 \mathrm{~mm}$ & 1.35 & $.83-2.19$ & .23 & 1.24 & $.68-2.26$ & .48 & 1.28 & $.77-2.13$ & .35 \\
\hline AUI device & 1.8 & $.98-3.37$ & .06 & 2.05 & $.99-4.26$ & .05 & 1.46 & $.79-2.73$ & .23 \\
\hline Baseline AAA diameter & 1.01 & $.99-1.02$ & .07 & 1.02 & $1.02-1.03$ & .02 & 1.01 & 1.01-1.02 & .03 \\
\hline Reverse-tapered & 1.26 & $.88-1.80$ & .21 & .82 & $.51-1.33$ & .43 & .86 & $.60-1.23$ & .41 \\
\hline
\end{tabular}

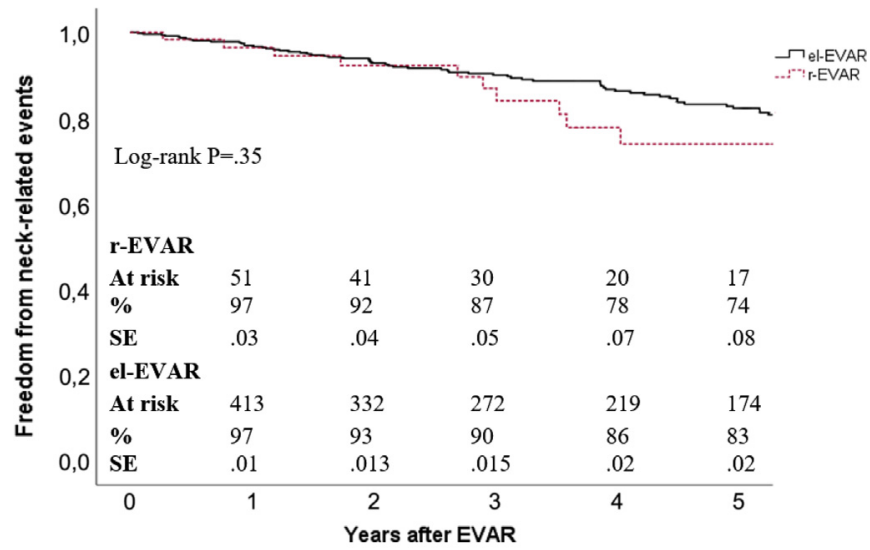

Fig 2. Kaplan-Meier curve for freedom from neck-related events between endovascular repair of a ruptured abdominal aortic aneurysm ( $r$-EVAR) and elective endovascular aneurysm repair (el-EVAR). Kaplan-Meier curves only include data from patients with two postoperative examinations. SE, Standard error.

underwent EVAR had a secondary intervention between 90 days and 3 years after EVAR. ${ }^{17}$ In the IMPROVE trial, most of the reinterventions were performed early in the postoperative period, and decreased during time. In our study, $12.3 \%$ of patients have been reintervened during index hospitalization, which is a slightly lower than found in the first 90 days of the IMPROVE trial (40 reinterventions in 29 of 185 patients). This little difference might easily be explained by the fact we only considered in-hospital period/30-day as early reintervention, whereas 90 days were considered in the IMPROVE trial. In favor of our argument, Broos et $\mathrm{al}^{6,17}$ found an even lower reintervention rate during hospitalization (3.4\%) compared with our study.

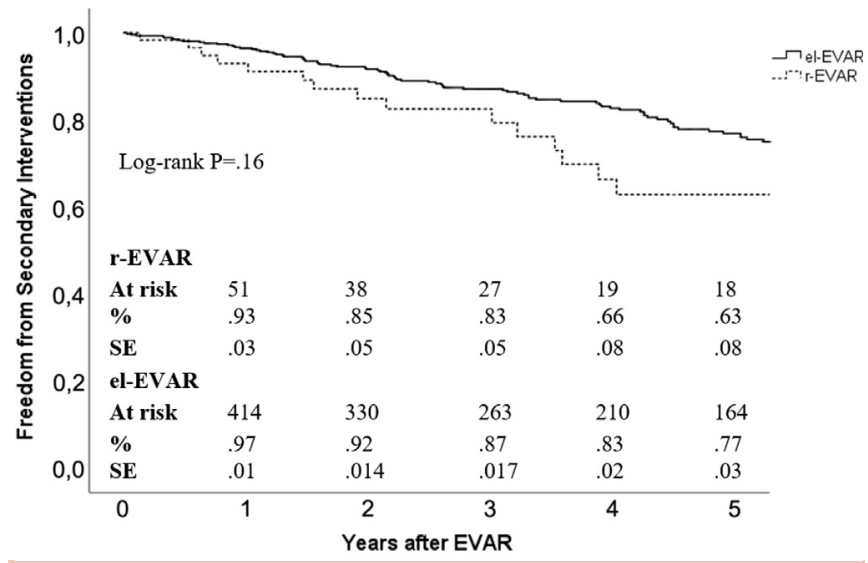

Fig 3. Kaplan-Meier curve for freedom from secondary interventions between endovascular repair of a ruptured abdominal aortic aneurysm ( $r$-EVAR) and elective endovascular aneurysm repair (el-EVAR). Kaplan-Meier curves only include data from patients with two postoperative examinations. SE, Standard error.

In our study, use outside the IFU was found to be predictive of secondary interventions, whereas the IMPROVE trial reported a relevant association with the iliac anatomy. ${ }^{17}$ For those patients developing complications in the IMPROVE trial, it would be relevant to analyze the anatomic characteristics leading to an increased risk of complications. Nevertheless, as with the IMPROVE trial, our study suggests that in selected patients, r-EVAR is able to achieve similar results up to midterm follow-up as if performed in intact AAAs.

In our study, r-EVAR patients were not at an increased risk of developing types I and III endoleaks when compared with el-EVAR patients despite the morphologically distinctly different anatomies, with the r-EVAR 


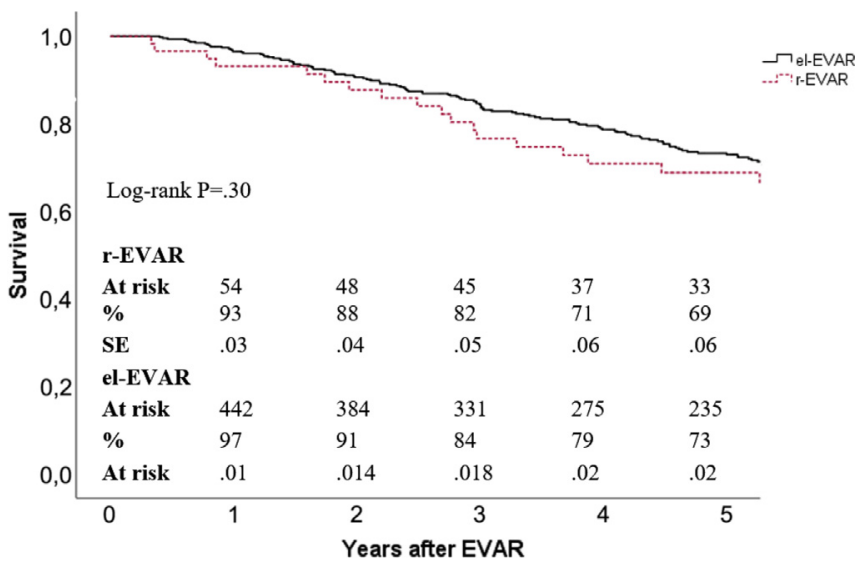

Fig 4. Kaplan-Meier curve of overall-survival after endovascular repair of a ruptured abdominal aortic aneurysm $(r-E V A R)$ and elective endovascular aneurysm repair (el-EVAR). Kaplan-Meier curves only include data from patients with two postoperative examinations. SE, Standard error.

group having significantly shorter and more angulated necks. Quinn et $\mathrm{al}^{7}(\mathrm{n}=2052)$ also found comparable rates of type l endoleaks between r-EVAR patients (5.4\%) and elEVAR ( $4.4 \% ; P=.68$ ) over a mean follow-up period of 30 months. Although the rates of type I endoleaks reported by Quinn et $\mathrm{al}^{7}$ are lower than the ones observed in our cohort, our population had a longer follow-up ( 4.3 years vs 2.5 years). In contrast, Broos et $\mathrm{al}^{6}$ found a significantly higher incidence of type I and III endoleaks among $r$-EVAR patients (21.3\% vs $10 \% ; P=.003$ ) at 5 years. The presence of more complex anatomies among r-EVAR patients combined with the emergency of the procedure may have increased likelihood for device IFU violation, which could explain the higher incidence of type I and III endoleaks in r-EVAR group, although this was not disclosed by those authors. ${ }^{18,19}$ In our study, we specifically addressed this question and found that patients treated outside the IFU were at greater risk for neck-related complications both in r-EVAR and el-EVAR groups. These data are corroborated by Baderkhan et al $^{19}$ in a multicenter study, including some of the patients figuring in our current report. Accordingly, graft-related complications were more common among r-EVAR patients treated outside IFU (30\%; 95\% Cl, 14\%-42\%) when compared with inside the IFU r-EVAR $6 \%$; $95 \% \mathrm{Cl}, 0 \%-13 \%$; $P=.015) .{ }^{19}$ Consequently, our data suggest that adequate sealing and IFU compliance rather than the timing of AAA repair are more important determinants of outcomes after EVAR. Furthermore, after being successful in obtaining initial exclusion of the ruptured AAA and survival of the patient, standard infrarenal r-EVAR when performed outside proximal neck IFU should regularly be considered as an intermediate step of a staged definitive repair, because these patients will have a similar life expectancy to el-EVAR patients.
Additionally, our data underline the evolution of standard aortic infrarenal endografts, with late generation devices outperforming older ones. ${ }^{20}$ Whereas in the study from Broos et al the Talent (Medtronic, Santa Rosa, Calif) was used in $63.3 \%$ of r-EVAR cases (vs 30.8\% of el-EVAR procedures) in our population the Endurant (Medtronic) was most commonly implanted device for r-EVAR. A comparative study from t'Mannetje et $\mathrm{al}^{27}$ found that the Talent was associated to an increased risk of proximal neck complications (odds ratio, 6.73; $95 \% \mathrm{Cl}, 1.65-27.4)$ when compared with the Endurant endoprosthesis as that device lacks active fixating such as hooks and barbs. These findings may also explain why $r$-EVAR patients had more type I and III endoleaks in the study by Broos et al. In our study, patients who died in hospital or were intraoperatively converted to open repair were excluded, which probably leaves out from the study a proportion of patients with a significantly hostile anatomy. Additionally, the Endurant stent graft was implanted in approximately $70 \%$ of $r$-EVAR group and the latter has the broadest IFU criteria. Furthermore, during study period approximately 50\% of rAAA had open repair. These reasons contributed for a relatively low incidence of patients outside IFU in our $r$-EVAR surviving population.

Aneurysm rupture represents the last stage of aortic aneurysm disease progression. Consequently, after excluding in-hospital mortality, a worse long-term survival might be anticipated in patients with a ruptured AAA when compared with patients treated for el-AAA. However, in our study, after discharge, long-term mortality was not significantly different among groups at 5 years, in agreement with previous reports. ${ }^{4}$ In fact, a similar burden of other competing cardiovascular diseases was found among groups, which may be a more ominous proxy of long-term survival than timing of repair.

There are several additional limitations that warrant consideration. First, the retrospective nature of our study, which is also from a single center, has the potential risk of selection bias. A randomized controlled trial would decreases the risk of bias, but would not entirely eliminate the procedure operator dependent and requires careful planning. The selection of patients with at least two postoperative examinations may give a biased estimate of survival, because patients have to survive for at least 6 months after EVAR; however, overall survival including these patients is provided in a supplementary analysis. Another limitation of this study was the difficulty in accessing the exact cause of death in all patients who died during the study period. These data were obtained from medical records, so caution is advised in the interpretation of the mortality-related data. Method of repair and type of device was left to the operator's choice. In addition, the lack of statistical power may have hampered the possibility to unveil subtler differences among patient groups. However, this study provides 
midterm data of ruptured and el-EVAR patients which may be combined with data from other studies and constitute relevant information for further future analysis.

\section{CONCLUSIONS}

Our study suggests that, after discharge, durable midterm outcomes results may be achieved in both elective and ruptured EVAR settings. Studies with a larger sample size and longer follow-up are needed to confirm safety of similar follow-up strategies in both groups.

\section{AUTHOR CONTRIBUTIONS}

Conception and design: JOP, RSF, NO, FG, AM, HV

Analysis and interpretation: JOP, RSF, NO, FG, SH, AM, HV

Data collection: JOP, RSF, NO, FG, MR, SR

Writing the article: JOP

Critical revision of the article: JOP, RSF, NO, FG, SH, MR,

SR, AM, HV

Final approval of the article: JOP, RSF, NO, FG, SH, MR, SR,

AM, HV

Statistical analysis: JOP, SH

Obtained funding: Not applicable

Overall responsibility: HV

\section{REFERENCES}

1. Gupta PK, Ramanan B, Engelbert TL, Tefera G, Hoch JR, Kent KC. A comparison of open surgery versus endovascular repair of unstable ruptured abdominal aortic aneurysms. J Vasc Surg 2014:60:1439-45.

2. Starnes BW, Quiroga E, Hutter C, Tran NT, Hatsumaki T, Meissner $M$, et al. Management of ruptured abdominal aortic aneurysm in the endovascular era. J Vasc Surg 2010;51: 9-17; discussion: 18.

3. Patel R, Sweeting MJ, Powell JT, Greenhalgh RM. Endovascular versus open repair of abdominal aortic aneurysm in 15-years' follow-up of the UK endovascular aneurysm repair trial 1 (EVAR trial 1): a randomised controlled trial. Lancet 2016;388:2366-74.

4. Mani K, Bjorck M, Lundkvist J, Wanhainen A. Improved long-term survival after abdominal aortic aneurysm repair. Circulation 2009;120:201-11.

5. Hinchliffe RJ, Alric P, Rose D, Owen V, Davidson IR, Armon MR, et al. Comparison of morphologic features of intact and ruptured aneurysms of infrarenal abdominal aorta. J Vasc Surg 2003;38:88-92.

6. Broos PP, t'Mannetje YW, Stokmans RA, Houtermans S, Corte G, Cuypers PW, et al. A 15-year single-center experience of endovascular repair for elective and ruptured abdominal aortic aneurysms. J Endovasc Ther 2016;23: 566-73.

7. Quinn AA, Mehta M, Teymouri MJ, Keenan ME, Paty PSK, Zhou $Y$, et al. The incidence and fate of endoleaks vary between ruptured and elective endovascular abdominal aortic aneurysm repair. J Vasc Surg 2017;65:1617-24.

8. Bastos Goncalves F, van de Luijtgaarden KM, Hoeks SE, Hendriks JM, ten Raa S, Rouwet EV, et al. Adequate seal and no endoleak on the first postoperative computed tomography angiography as criteria for no additional imaging up to 5 years after endovascular aneurysm repair. J Vasc Surg 2013;57:1503-11.

9. Baderkhan $\mathrm{H}$, Haller O, Wanhainen A, Bjorck M, Mani K. Follow-up after endovascular aortic aneurysm repair can be stratified based on first postoperative imaging. Br J Surg 2018:105:709-18.

10. Bastos Goncalves F, Jairam A, Voute MT, Moelker AD, Rouwet EV, ten Raa S, et al. Clinical outcome and morphologic analysis after endovascular aneurysm repair using the Excluder endograft. J Vasc Surg 2012;56:920-8.

11. van Keulen JW, Moll FL, Tolenaar JL, Verhagen HJ, van Herwaarden JA. Validation of a new standardized method to measure proximal aneurysm neck angulation. J Vasc Surg 2010;51:821-8.

12. van Prehn J, van der Wal MB, Vincken K, Bartels LW, Moll FL, van Herwaarden JA. Intra- and interobserver variability of aortic aneurysm volume measurement with fast CTA postprocessing software. J Endovasc Ther 2008;15: 504-10.

13. Balm R, Stokking R, Kaatee R, Blankensteijn JD, Eikelboom BC, van Leeuwen MS. Computed tomographic angiographic imaging of abdominal aortic aneurysms: implications for transfemoral endovascular aneurysm management. J Vasc Surg 1997:26:231-7.

14. Chaikof EL, Blankensteijn JD, Harris PL, White GH, Zarins CK, Bernhard VM, et al. Reporting standards for endovascular aortic aneurysm repair. J Vasc Surg 2002;35:1048-60.

15. Bastos Goncalves $F$, Baderkhan $H$, Verhagen $H J$, Wanhainen A, Bjorck M, Stolker RJ, et al. Early sac shrinkage predicts a low risk of late complications after endovascular aortic aneurysm repair. Br J Surg 2014;101:802-10.

16. Comparative clinical effectiveness and cost effectiveness of endovascular strategy $v$ open repair for ruptured abdominal aortic aneurysm: three year results of the IMPROVE randomised trial. BMJ (Clinical Res Ed) 2017;359:j4859.

17. Powell JT, Sweeting MJ, Ulug P, Thompson MM, Hinchliffe RJ. Editor's choice - re-interventions after repair of ruptured abdominal aortic aneurysm: a report from the IMPROVE Randomised Trial. Eur J Vasc Endovasc Surg 2018;55:625-32.

18. Herman CR, Charbonneau P, Hongku K, Dubois L, Hossain S, Lee $\mathrm{K}$, et al. Any nonadherence to instructions for use predicts graft-related adverse events in patients undergoing elective endovascular aneurysm repair. J Vasc Surg 2018;67: 126-33.

19. Baderkhan $H$, Goncalves FM, Oliveira NG, Verhagen $H J$, Wanhainen A, Bjorck M, et al. Challenging anatomy predicts mortality and complications after endovascular treatment of ruptured abdominal aortic aneurysm. J Endovasc Ther 2016:23:919-27.

20. Tadros RO, Faries PL, Ellozy SH, Lookstein RA, Vouyouka AG, Schrier R, et al. The impact of stent graft evolution on the results of endovascular abdominal aortic aneurysm repair. J Vasc Surg 2014:59:1518-27.

21. t'Mannetje YW, Cuypers PW, Saleem BR, Bode AS, Teijink JA, van Sambeek MR. Comparison of midterm results for the Talent and Endurant stent graft. J Vasc Surg 2017;66:735-42.

Submitted Mar 14, 2019; accepted Jul 3, 2019.

Additional material for this article may be found online at www.jvascsurg.org. 
Journal of Vascular Surgery

Volume a, Number

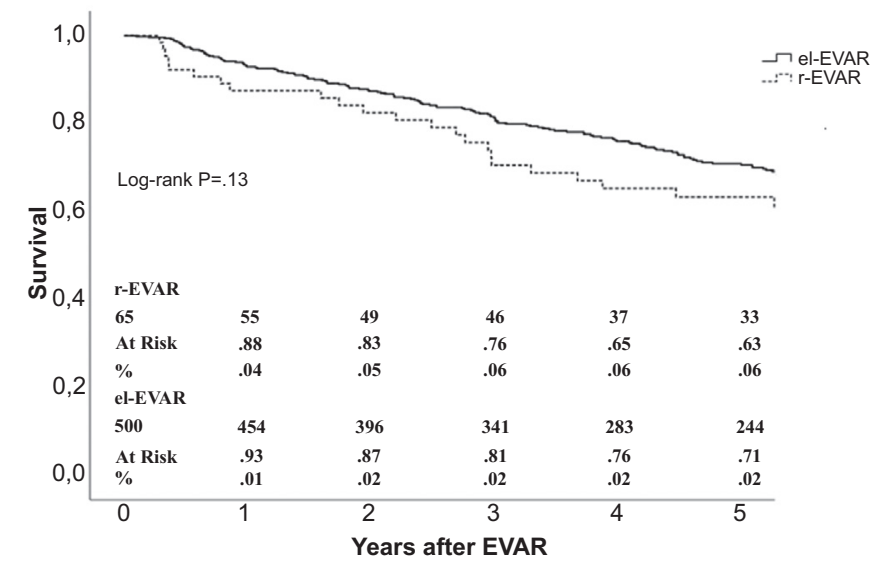

Supplementary Fig (online only). Kaplan-Meier curve of overall-survival after endovascular repair of a ruptured abdominal aortic aneurysm ( $r$-EVAR) and elective endovascular aneurysm repair (el-EVAR), including patients previously excluded owing to a lack of two postoperative examinations. SE, Standard error. 